\title{
Maneuvering target tracking using fuzzy logic-based recursive least squares filter
}

\author{
En Fan ${ }^{1 *}$, Wei-xin $\mathrm{Xie}^{2}$ and Zong-xiang Liu ${ }^{2}$
}

\begin{abstract}
In this paper, a fuzzy logic-based recursive least squares filter (FLRLSF) is presented for maneuvering target tracking (MTT) in situations of observations with unknown random characteristics. In the proposed filter, fuzzy logic is applied in the standard recursive least squares filter (RLSF) by the design of a set of fuzzy if-then rules. Given the observation residual and the heading change in the current prediction, these rules are used to determine the magnitude of the fading factor of RLSF. The proposed filter has an advantage in which the restrictive assumptions of statistical models for process noise, measurement noise, and motion models are relaxed. Moreover, it does not need a maneuver detector when tracking a maneuvering target. The performance of FLRLSF is evaluated by using a simulation and real test experiment, and it is found to be better than those of the traditional RLSF, the fuzzy adaptive a- $\beta$ filter (FAa- $\beta F$ ), and the hybrid Kalman filter in tracking accuracy.
\end{abstract}

Keywords: Maneuvering target tracking; Adaptive filter; Recursive least squares filter; Fading factor; Fuzzy logic; Heading change

\section{Introduction}

Maneuvering target tracking (MTT) is always a critical problem in target tracking area [1-5]. In the literature on MTT in sensor network, its survey primarily consists of target dynamic models, observation models and techniques, decision-based methods, multiple-model methods, and nonlinear filtering methods. The adaptive filters are popular to apply in MTT as a nonlinear-type filter; they are mainly classified as follows [2]: structured adaptive filters, such as the interacting multiple model (IMM) and the variable structured model; parametric adaptive filters, such as the Singer model, the current statistical model, and the adaptive acceleration model. Structured adaptive filters, usually with more computation, require sufficient prior knowledge such as all possible motion models of a moving target. So, they are less suitable for real situations with limited prior knowledge. Parametric adaptive filters, generally with less computation, describe maneuver characteristics as unknown random parameters with certain probability distribution functions (pdf) and estimate maneuver models jointly by both the parameters and the target

\footnotetext{
* Correspondence: efan@stu.xidian.edu.cn

${ }^{1}$ School of Electronic Engineering, Xidian University, Xi'an, Shanxi 710071, China

Full list of author information is available at the end of the article
}

states [2]. Recently, particle filters are broadly adopted in various applications, such as location estimation [6-8]. However, their tracking performances are combined in proportion to the number of the corresponding particles in target tracking. Moreover, the computation of these filters is generally complicated. Hence, they are unsuitable to apply in real-time tracking [9-11]. In real sensor networks, observations or state estimates are generally transmitted without their covariances. They are processed in sensor nodes or fusion centers. To relax transmission burdens and save communication bandwidths, the filters designed need to possess good performances in both computational and time complexity. For the above reasons, this paper is only concerned with parametric adaptive filters.

Maneuver modeling which is a usual prerequisite for MTT has a direct influence on tracking results [5]. Several techniques have been introduced to overcome different maneuver modeling. MTT methods based on statistical models often need to establish motion models exactly and obtain the target position timely. Unfortunately, these requirements are difficult to satisfy because of unknown maneuver characteristics in real situations. To solve the problem, one of the most popular techniques has been applied in MTT. Fuzzy logic with 
intelligent adaptation capabilities has been widely used to relax or avoid the restrictive assumptions of motion models [12-16]. Due to the limitation of the standard IMM algorithm in real applications, the adaptive fuzzy IMM filter (AFIMMF) proposed in [12] defines several basis sub-models and time-varying mode transition probabilities to reduce its computational complexity. Nevertheless, it suffers from a deficiency originated in expensive computation of the time-varying mode transition probabilities. Moreover, its performance depends on the assumptions on the basis sub-models. Two different modified Kalman filters proposed in [13] and [14] tend to extend the standard Kalman filter for MTT. Nonetheless, their computation is still expensive, and the proposed hybrid Kalman filter (HKF) in [14] is mainly applied to track an accelerating target. To track a target making sharp turns or accelerating at nonuniform rates in stressful environments, the fuzzy gain $\alpha-\beta$ filter (FG $\alpha-$ $\beta F)$ is proposed in [15]. It can avoid any assumptions on statistical models by utilizing fuzzy rules to determine the magnitudes of $\alpha$ and $\beta$. However, its tracking result in the case where sharp turns appear is not improved markedly. For perfect tracking accuracy, the fuzzy adaptive $\alpha-\beta$ filter $(F A \alpha-\beta F)$ proposed in [16] can detect maneuvers. However, its tracking result is still undesirable.

In practice, the standard recursive least squares filter (RLSF) $[17,18]$ is quite well received in linear models with the unknown random characteristics of observations. In this case, it has less computational complexity than the standard Kalman filter. Unfortunately, its performance degrades seriously during maneuvering. For perfect output results in nonlinear models, the traditional fuzzy adaptive filters make the relationship between the input and output variables maps into a set of fuzzy rules, and these rules determine the control parameters of the filters. So, their performances depend on whether the rules can describe maneuver characteristics exactly and completely. Due to the unknown motion models during real tracking procedures, a complex nonlinear relationship exists between observations and state estimates. For this reason, the fuzzy rules are usually difficult to establish by directly utilizing the relationship between the observations and the state estimates. Therefore, the traditional fuzzy adaptive filters are restricted in the applications of MTT.

Applying fading factors and fuzzy logic into traditional filters are popular because of some practical concerns including easy implementation and effectiveness. In this respect, it provides a direction to design a filter for MTT [19]. Considering these facts, a novel fuzzy logic-based recursive least squares filter (FLRLSF) is proposed to deal with the problem mentioned above. First, the proposed filter utilizes observation residuals and heading changes to describe maneuver characteristics of a maneuvering target and employs the fading factor of RLSF to express the magnitude of the target maneuver. A set of fuzzy rules is designed according to the relationship of the fading factor with observation residuals and heading changes over time. Next, given the observation residual and the heading change in the current prediction, these rules are applied to determine the magnitude of the fading factor. Then, the fuzzy system designed based on the rules is utilized to adjust the fading factor in response to the changes in speed and direction without maneuver detectors. Finally, FLRLSF is applied to estimate the target state. Its performance is evaluated by using a simulation and real test experiment, compared with the existing filters mentioned above.

\section{Analysis of maneuvering target motions}

Target motion models form two categories, the linear and maneuver (nonlinear) models. In linear models, the motion and observation model of a moving target are defined as follows:

$$
\begin{aligned}
& \boldsymbol{x}_{k+1}=\Phi_{k} \boldsymbol{x}_{k}+\boldsymbol{v}_{k} \\
& \boldsymbol{z}_{k}=H_{k} \boldsymbol{x}_{k}+\boldsymbol{w}_{k}
\end{aligned}
$$

where $\boldsymbol{x}_{k}$ denotes an $n$-dimensional state vector about the target at time $k$ and $z_{k}$ denotes an $m$-dimensional observation vector. $\Phi_{k}$ is an $n \times n$ state transition matrix, and $H_{k}$ is an $m \times n$ observation transition matrix. The process noise $\boldsymbol{v}_{k}$ is assumed to be Gaussian white noise with zero mean and covariance $Q_{k}$, while the observation noise $\boldsymbol{w}_{k}$ is assumed to be the zero-mean Gaussian noise with the covariance $R_{k}$.

$$
\begin{aligned}
& E\left[v_{k} v_{t}\right]=Q_{k} \delta_{k t} \\
& E\left[w_{k} w_{t}\right]=R_{k} \delta_{k t}
\end{aligned}
$$

where is $\delta_{k t}$ the Kronecker delta function.

Though targets often move at constant velocity, they are easy to maneuver suddenly. In this respect, maneuver modeling is a key problem of MTT. To solve this problem, many methods have been proposed on the restrictive assumptions of the motion models $[17,20]$. Considering the aforementioned facts, these assumptions are often inconsistent with real environments. Then, two strategies of motion modeling exist with unknown maneuver information: describe the target trajectory as several typical motion models with known parameters or their combination [3] or incorporate control variables in the target motion equation as the random variables with the certain pdf [20]. Unfortunately, it is difficult to obtain the prior information in real situations, and various uncertainties generally exist in maneuver motions during real tracking procedures [16]. So, the methods based on the statistic framework are complicated and difficult due 
to establishing the accurate motion models or obtaining the exact pdf by using the probability and statistics theory. Nowadays, fuzzy systems with the universal approximation capabilities have been widely applied in nonlinear complicated system [21,22]. Incorporation of fuzzy logic in fuzzy systems is easy and flexible to describe various uncertainties or random variables by the use of linguistic terms. From this point of view, it is a good idea that fuzzy systems are designed to adjust the filter parameter for MTT.

In addition, observations from a target contain much information of kinematics characteristics, whether the target maneuvers or moves at constant velocity. Theoretically, applying characteristic information into filter estimation can help the maneuvering modeling and improve the performances of tracking results. In various characteristics, observation residuals and heading changes are two important characteristics of the motion model. They can perfectly reflect the relationship between observations and maneuvers. On the one hand, if observation residual or heading change is large, the target is strongly maneuvering with high probability. On the other hand, if the observation residual or heading change is small, the target is weakly maneuvering with high probability. In addition, incorporation of them in tracking methods may lead to an immediate detection and a less delay about maneuvers $[2,14,16]$. Considering this fact, observation residuals and heading changes are usually combined to detect maneuvers. They can be calculated with the following relations:

$$
\begin{aligned}
\Delta z_{k} & =\left[\left(\boldsymbol{z}_{k}-H_{k} \Phi_{k} \hat{\boldsymbol{x}}_{k-1}\right)^{\mathrm{T}}\left(\boldsymbol{z}_{k}-H_{k} \Phi_{k} \hat{\boldsymbol{x}}_{k-1}\right)\right]^{1 / 2} \\
\Delta \theta_{k} & =\left|\varphi_{k}-\phi_{k-1}\right|
\end{aligned}
$$

where $\Delta z_{k}$ and $\Delta \theta_{k}$ denote observation residual and heading change; $\phi_{k}$ is the angle between the observation vector $z_{k}$ and the state estimate vector, while $\phi_{k-1}$ is the angle between the state estimate vector and the state estimate vector; and the superscript $\mathrm{T}$ denotes transpose. Here, $\varphi_{k}$ and $\phi_{k-1}$ are obtained from the following forms:

$$
\begin{aligned}
& \varphi_{k}=\arctan \left[\left(y_{k}-\hat{y}_{k-1}\right) /\left(x_{k}-\hat{x}_{k-1}\right)\right] \\
& \phi_{k-1}=\arctan \left[\left(\hat{y}_{k-1}-\hat{y}_{k-2}\right) /\left(\hat{x}_{k-1}-\hat{x}_{k-2}\right)\right]
\end{aligned}
$$

where $x_{k}, \hat{x}_{k-1}$, and $\hat{x}_{k-2}$ are the components of $\boldsymbol{z}_{k}, \hat{\boldsymbol{x}}_{k-1}$, and $\hat{\boldsymbol{x}}_{k-2}$ in the $x$-axis direction respectively, while $y_{k}$, $\hat{y}_{k-1}$, and $\hat{y}_{k-2}$ are their corresponding components in the $y$-axis direction. To simplify both computation and discussion, $\Delta z_{k}$ and $\Delta \theta_{k}$ are necessary in normalization processing with the following forms: $\Delta z_{k}{ }^{\prime}=\Delta z_{k} / \Delta z_{\max }$ and $\Delta \theta_{k}{ }^{\prime}=\Delta \theta_{k} / \Delta \theta_{\max }$ instead of $\Delta z_{k}$ and $\Delta \theta_{k}$. Here, $\Delta z_{\max }$ and $\Delta \theta_{\max }$ are their corresponding maximum values, and they are usually related with the target's type and the sensor's performance.

\section{Traditional RLSF method}

RLSF is an effective estimate method in a linear motion model, and it is widely used to estimate the target state in target tracking $[17,18]$. Using Equation 2, one can obtain at time $k$

$$
\boldsymbol{z}^{k}=H^{k} \boldsymbol{x}_{k}+\boldsymbol{w}^{k}
$$

where $z^{k}=\left(z_{1}, z_{2}, \ldots, z_{k}\right)^{\mathrm{T}}, H^{k}=\left(H_{1}, H_{2}, \ldots, H_{k}\right)^{\mathrm{T}}$, and $\boldsymbol{w}^{k}=$ $\left(\boldsymbol{w}_{1}, \boldsymbol{w}_{2}, \ldots, \boldsymbol{w}_{k}\right)^{\mathrm{T}}$.

Give the quadratic error function $C_{k}$ as follows:

$$
C_{k}=\left(\boldsymbol{z}^{k}-H^{k} \boldsymbol{x}_{k}\right)^{\mathrm{T}} F_{k}\left(\boldsymbol{z}^{k}-H^{k} \boldsymbol{x}_{k}\right)
$$

where $F_{k}$ is a diagonal matrix, whose main diagonal elements are $\lambda_{k}^{k-1}, \ldots, \lambda_{k}, 1$. Here, $\lambda_{k}$ is called the fading factor lied in $(0,1]$. The least squares estimate vector equals the corresponding value $\boldsymbol{x}_{k}$ when $C_{k}$ obtains its minimum value. To obtain $\hat{\boldsymbol{x}}_{k}$, the function (10) is differentiated for $\boldsymbol{x}_{k}$ to yield

$$
\nabla_{\boldsymbol{x}_{k}} C_{k}=\frac{\partial}{\partial \boldsymbol{x}_{k}}\left(\boldsymbol{z}^{k}-H^{k} \boldsymbol{x}_{k}\right)^{\mathrm{T}} F_{k}\left(\boldsymbol{z}^{k}-H^{k} \boldsymbol{x}_{k}\right)
$$

so that $\hat{\boldsymbol{x}}_{k}$ is derived by solving Equation 11 for $\boldsymbol{x}_{k}$

$$
\hat{\boldsymbol{x}}_{k}=P_{k}\left(H^{k}\right)^{\mathrm{T}} F_{k} z^{k}
$$

where $P_{k}$ is the estimate error covariance at time $k$, calculated in the following form:

$$
P_{k}=\left[\left(H^{k}\right)^{\mathrm{T}} F_{k} H^{k}\right]^{-1}
$$

As a result, Equations 12 and 13 can be rewritten in the recursive formularies, respectively,

$$
\begin{aligned}
\hat{\boldsymbol{x}}_{k}= & \Phi_{k} \hat{\boldsymbol{x}}_{k-1}+P_{k} H_{k}^{\mathrm{T}}\left(\boldsymbol{z}_{k}-H_{k} \Phi_{k} \hat{\boldsymbol{x}}_{k-1}\right) \\
P_{k}= & \lambda_{k}^{-1} \Phi_{k} P_{k-1} \Phi_{k}^{\mathrm{T}}-\lambda_{k}^{-2} \Phi_{k} P_{k-1} \Phi_{k}^{\mathrm{T}} H_{k}^{\mathrm{T}} \\
& \times\left(I+\lambda_{k}^{-1} H_{k} \Phi_{k} P_{k-1} \Phi_{k}^{\mathrm{T}} H_{k}^{\mathrm{T}}\right)-1 H_{k} \Phi_{k} P_{k-1} \Phi_{k}^{\mathrm{T}}
\end{aligned}
$$

for $k \geq 3$. Equations 14 and 15 are called RLSF for $\boldsymbol{x}_{k}$ [18]. The initial state is determined with the observations $z_{1}$ and $z_{2}$ at time $k=1,2$ as follows:

$$
\begin{aligned}
& \hat{\boldsymbol{x}}_{2}=P_{2}\left(H^{2}\right)^{\mathrm{T}} z^{2} \\
& P_{2}=\left[\left(H^{2}\right)^{\mathrm{T}} H^{2}\right]^{-1}
\end{aligned}
$$

From Equations 14 and 15, $\hat{\boldsymbol{x}}_{k}$ is the sum of the state prediction and the deviation between the observation and the predicted position at time $k$ on the one hand, and the magnitude of $\hat{\boldsymbol{x}}_{k}$ depends on how large the 
deviation is modified by $P_{k}$ on the other hand. Meanwhile, $P_{k}$ can vary with $\lambda_{k}$. From this point of view, $\hat{\boldsymbol{x}}_{k}$ can be indirectly modified by adjusting $\lambda_{k}$. While the modification of $\hat{\boldsymbol{x}}_{k}$ becomes weaker with the increase of $\lambda_{k}$, it becomes greater with the decrease of $\lambda_{k}$. In this respect, if one can obtain the right $\lambda_{k}$ according to maneuver information, it implies that $\hat{\boldsymbol{x}}_{k}$ can be exactly expressed as the modified value by adjusting $\lambda_{k}$ at each discrete time. Therefore, RLSF can be applied to estimate the target state by employing $\lambda_{k}$ to describe maneuver characteristics.

\section{FLRLSF method for MTT}

As the results in Sections 2 and 3 show, $\Delta z_{k}{ }^{\prime}$ and $\Delta \theta_{k}{ }^{\prime}$ can be combined to detect maneuvers, and $\lambda_{k}$ can be employed to describe maneuver characteristics. Considering this fact, FLRLSF can be applied in MTT. It is illustrated in Figure 1. In the figure, block 1 calculates $\Delta z_{k}{ }^{\prime}$ and $\Delta \theta_{k}{ }^{\prime}$ based on $z_{k}$ and $\hat{\boldsymbol{x}}_{k-1}$. Blocks 2 to 5 compose a fuzzy system to obtain the optimum $\tilde{\lambda}_{k}$, elaborated in Section 4.1. Block 6 is RLSF, which can obtain the optimal state estimate $\hat{\boldsymbol{x}}_{k}$ by the optimum $\tilde{\lambda}_{k}$ calculated by the fuzzy system. For clarity and perception, the fading factor is defined as a fuzzy variable, viz. replacing $\lambda_{k}$ with $\tilde{\lambda}_{k}$. Here, the main functions of the fuzzy system give as follows: first, map the relationship of $\tilde{\lambda}_{k}$ with $\Delta z_{k}{ }^{\prime}$ and $\Delta \theta_{k}{ }^{\prime}$ into a set of fuzzy rules; then, according to these rules, take $\Delta z_{k}{ }^{\prime}$ and $\Delta \theta_{k}{ }^{\prime}$ as the input variables of the fuzzy system and express its output variable as the value of $\tilde{\lambda}_{k}$; finally, estimate the target state by adjusting $\tilde{\lambda}_{k}$ to modify $\hat{\boldsymbol{x}}_{k}$.

\subsection{Design of the fuzzy system}

As shown in Figure 1, the design procedure of the fuzzy system, which consists of four blocks, includes the following: first, utilize a singleton fuzzifier as block 2; next, design the fuzzy rules of block 3 according to the relationship between the input and output variable of block 4 ; then, determine the reference engine of block 4, which maps the spaces of all variables into the fuzzy spaces and gives their membership functions; finally, select a defuzzifier of block 5. Each step is elaborated in detail as follows.

The values of two input variables are mapped into four fuzzy sets, respectively, which are expressed as $\tilde{A}_{i}$ and $\tilde{B}_{j}$, $i, j=1,2,3,4$, defined in the universe of $\Delta z_{k}{ }^{\prime}$ and $\Delta \theta_{k}{ }^{\prime}$. These fuzzy sets are labeled in the linguistic terms of zero (ZE), small positive (SP), medium positive (MP), and large positive (LP). To ensure the probability of each input data point of $\Delta z_{k}{ }^{\prime}$ or $\Delta \theta_{k}{ }^{\prime}$ falling into each fuzzy set be roughly equal, each fuzzy set is assigned to an interval of equal width and similar rules. In addition, triangular functions can simplify the computation of fuzzy reference engines and suppress the noise of input variables. So, they are used to define the membership functions of $\Delta z_{k}{ }^{\prime}$ or $\Delta \theta_{k}{ }^{\prime}$ in the corresponding universe for each fuzzy rule shown in Figure 2. The output consequence of the fuzzy system is expressed as the value of $\tilde{\lambda}_{k}$. It turns out that the performance of RLSF for MTT is more comfortable when the optimum $\tilde{\lambda}_{k}$ is found more often in the interval $(0,0.3]$. Based on the fact, to obtain the optimum $\tilde{\lambda}_{k}$, the region of $\tilde{\lambda}_{k}$ is divided into six different fuzzy sets, ZE, SP, MP, VP, large positive (LP), and extremely large positive (EP), expressed as $\tilde{C}_{m}, m=1,2, \ldots, 6$. The membership function

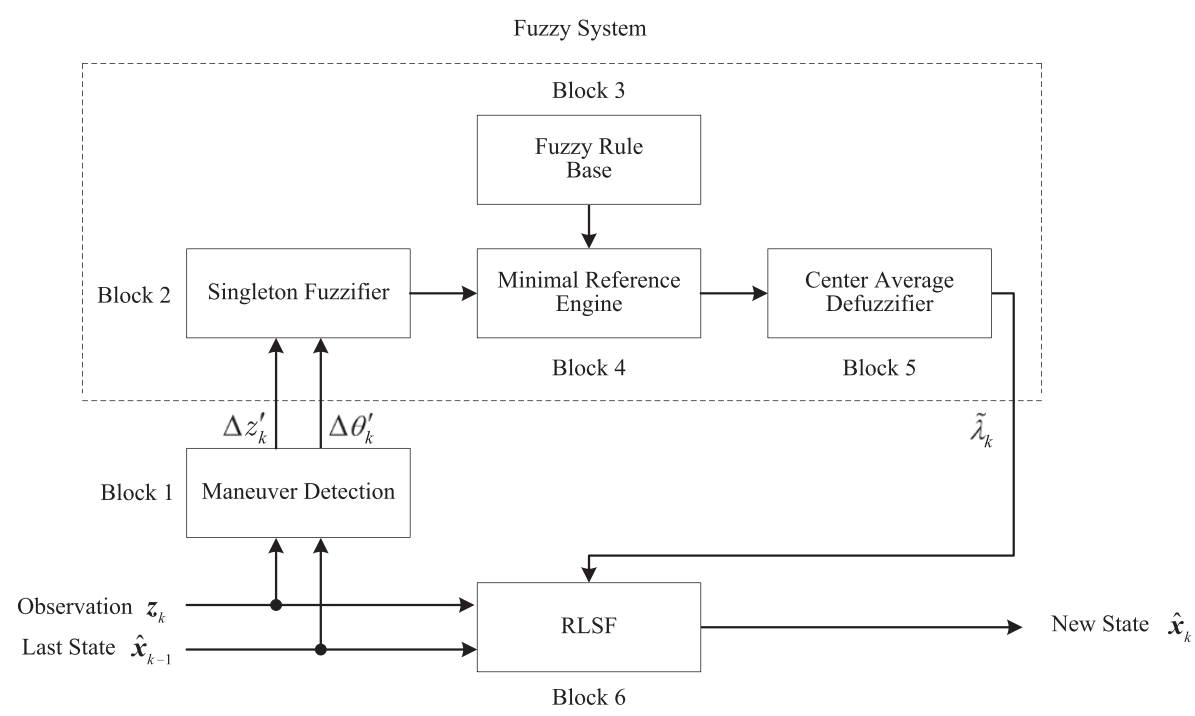

Figure 1 FLRLSF method. 


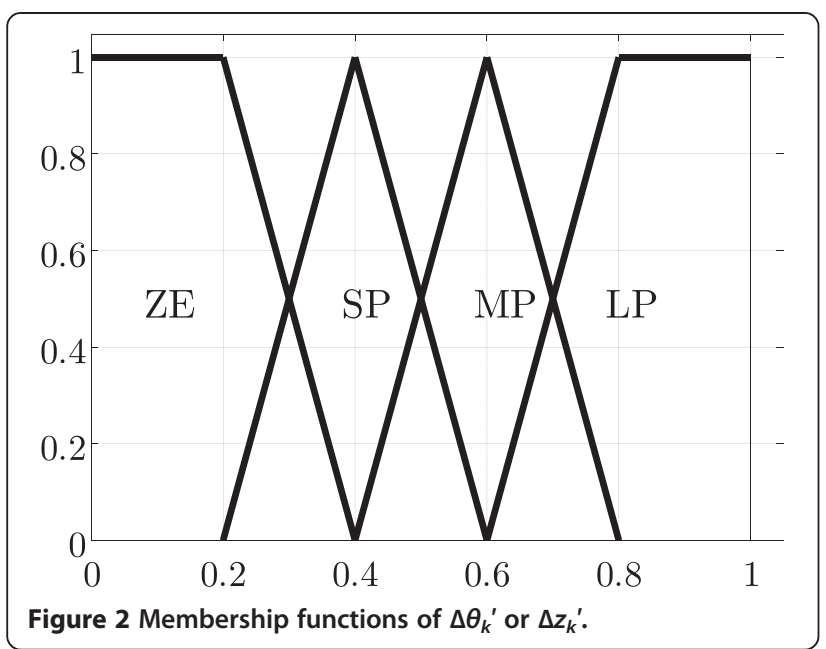

of $\Delta z_{k}{ }^{\prime}$ in each fuzzy set is determined in the following formula:

$$
\mu_{\tilde{A}_{i}^{l}}\left(\Delta z_{k}^{\prime}\right)=1-\frac{\left|\Delta z_{k}^{\prime}-c_{i}^{l}\right|}{b_{i}^{l}}, \Delta z_{k}^{\prime} \in\left[c_{i}^{l}-b_{i}^{l}, c_{i}^{l}+b_{i}^{l}\right]
$$

where $b_{i}^{l}$ and $c_{i}^{l}$ are the control parameters. The membership functions of $\Delta \theta_{k}{ }^{\prime}$ and $\tilde{\lambda}_{k}$ are similarly defined as $\Delta z_{k}{ }^{\prime}$, as shown in Figures 2 and 3 , respectively.

Utilize the fuzzy rules to establish the fuzzy model of the relationship of $\tilde{\lambda}_{k}$ with $\Delta z_{k}{ }^{\prime}$ and $\Delta \theta_{k}{ }^{\prime}$ :

$$
R^{l}: \text { IF } \Delta z_{k}^{\prime} \in \tilde{A}_{i}^{l} \text { AND } \Delta \theta_{k}^{\prime} \in \tilde{B}_{j}^{l} \text { THEN } \tilde{\lambda}_{k} \in \tilde{C}_{m}^{l}
$$

where $i, j=1,2,3,4, l=1,2, \ldots, M$, and $M=16$. The requirement for adjusting $\tilde{\lambda}_{k}$ is given below: if $\Delta z_{k}{ }^{\prime}$ or $\Delta \theta_{k}{ }^{\prime}$ increases, then it indicates that the maneuver gets stronger and $\tilde{\lambda}_{k}$ needs to be set relatively small; otherwise, if $\Delta z_{k}{ }^{\prime}$ or $\Delta \theta_{k}{ }^{\prime}$ decreases, then it indicates that the maneuver

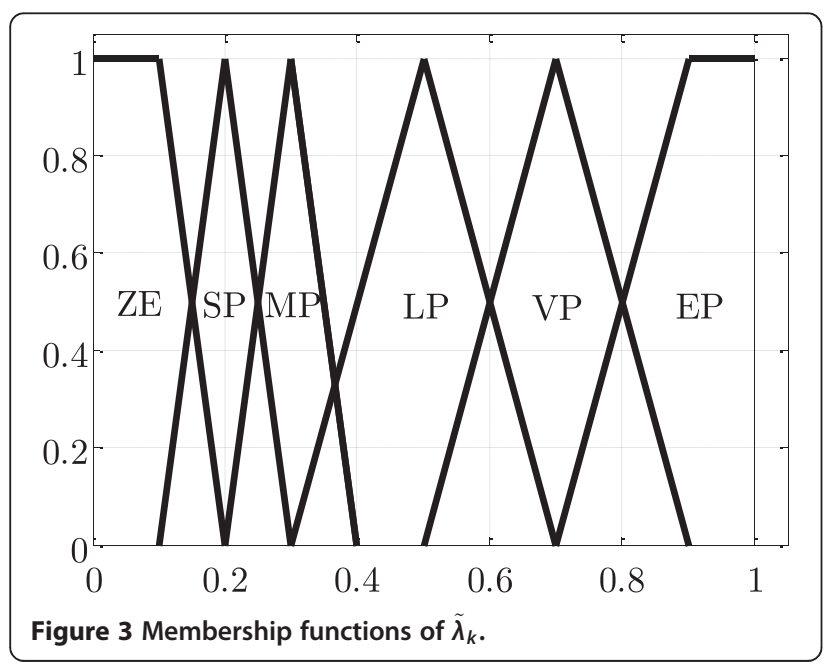

gets weaker and $\tilde{\lambda}_{k}$ needs to be set relatively large. The fuzzy rule base is designed as shown in Table 1 .

Due to less computation and more explicitness of both the minimal reference engine and the center average defuzzier, they are usually combined to establish a fuzzy system. The minimal reference engine and the center average defuzzier are expressed in the following forms [21]:

$$
\begin{aligned}
& \mu_{\tilde{C}_{m}^{l}}\left(\bar{\lambda}_{k}^{l}\right)=\min \left(\mu_{\tilde{A}_{i}^{l}}\left(\Delta z_{k}^{\prime}\right), \mu_{\tilde{B}_{j}^{l}}\left(\Delta \theta_{k}^{\prime}\right)\right) \\
& \tilde{\lambda}_{k}=\frac{\sum_{l=1}^{M} \bar{\lambda}_{m}^{l} \mu_{\tilde{C}_{m}}^{l}\left(\bar{\lambda}_{k}^{l}\right)}{\sum_{l=1}^{M} \mu_{\tilde{C}_{m}}^{l}\left(\bar{\lambda}_{k}^{l}\right)}
\end{aligned}
$$

As a result, the fuzzy system is given as follows:

$$
f\left(\Delta z_{k}^{\prime}, \Delta \theta_{k}^{\prime}\right)=\frac{\sum_{l=1}^{M} \bar{\lambda}_{m}^{l}\left[\min \left(\mu_{\tilde{A}_{i}^{l}}\left(\Delta z_{k}^{\prime}\right), \mu_{\tilde{B}_{j}^{l}}\left(\Delta \theta_{k}^{\prime}\right)\right)\right]}{\sum_{l=1}^{M}\left[\min \left(\mu_{\tilde{A}_{i}^{l}}\left(\Delta z_{k}^{\prime}\right), \mu_{\tilde{B}_{j}^{l}}\left(\Delta \theta_{k}^{\prime}\right)\right)\right]}
$$

where $\mu_{\tilde{A}_{i}^{l}}, \mu_{\tilde{B}_{j}^{l}}$, and $\mu_{\tilde{C}_{m}}^{l}$ denote the membership functions of $\Delta z_{k}^{\prime},{ }^{\prime} \Delta \theta_{k}^{\prime}$, and $\tilde{\lambda}_{k}$, respectively; $\bar{\lambda}_{k}^{l}$ at each discrete time is equivalent to the corresponding value of $\tilde{\lambda}_{k}$ when the membership function of $\tilde{\lambda}_{k}$ is maximized at each fuzzy set defined on.

\subsection{FLRLSF method}

As described at the beginning of Section 4, FLRLSF is able to detect the target maneuver and estimate its state by the fuzzy system. Based on the above conclusions, the recursive form of FLRLSF is expressed as

$$
\begin{aligned}
\hat{\boldsymbol{x}}_{k}= & \Phi_{k} \hat{\boldsymbol{x}}_{k-1}+P_{k} H_{k}^{\mathrm{T}}\left(\boldsymbol{z}_{k}-H_{k} \Phi_{k} \hat{\boldsymbol{x}}_{k-1}\right) \\
P_{k}= & \tilde{\lambda}_{k}^{-1} \Phi_{k} P_{k-1} \Phi_{k}^{\mathrm{T}}-\tilde{\lambda}_{k}^{-2} \Phi_{k} P_{k-1} \Phi_{k}^{\mathrm{T}} H_{k}^{\mathrm{T}} \\
& \times\left(I+\tilde{\lambda}_{k}^{-1} H_{k} \Phi_{k} P_{k-1} \Phi_{k}^{\mathrm{T}} H_{k}^{\mathrm{T}}\right)-1 H_{k} \Phi_{k} P_{k-1} \Phi_{k}^{\mathrm{T}}
\end{aligned}
$$

Table 1 Fuzzy rules on $\Delta \theta_{k^{\prime}}, \Delta z_{k^{\prime}}$, and $\tilde{\lambda}_{k}$

\begin{tabular}{llllll}
\hline & & $\Delta \boldsymbol{\theta}_{\boldsymbol{k}}{ }^{\prime}$ & & & \\
\cline { 3 - 6 } & & ZE & SP & MP & LP \\
\hline$\Delta z_{k}{ }^{\prime}$ & ZE & EP & EP & LP & MP \\
& SP & EP & VP & MP & SP \\
& MP & LP & MP & SP & ZE \\
& LP & MP & SP & ZE & ZE \\
\hline
\end{tabular}


where

$$
\tilde{\lambda}_{k}=\frac{\sum_{l=1}^{M} \bar{\lambda}_{m}^{l} \sup _{\Delta z_{k}^{\prime} \in \tilde{A}_{i}^{l}, \Delta \theta_{k}^{\prime} \in \tilde{B}_{j}^{l}} \min \left(\mu_{\tilde{A}_{i}^{l}}\left(\Delta z_{k}^{\prime}\right), \mu_{\tilde{B}_{j}^{l}}\left(\Delta \theta_{k}^{\prime}\right)\right)}{\sum_{l=1}^{M} \sup _{\Delta z_{k}^{\prime} \in \tilde{A}_{i}^{l}, \Delta \theta_{k}^{\prime} \in \tilde{B}_{j}^{l}} \min \left(\mu_{\tilde{A}_{i}^{l}}\left(\Delta z_{k}^{\prime}\right), \mu_{\tilde{B}_{j}^{l}}\left(\Delta \theta_{k}^{\prime}\right)\right)}
$$

The FLRLSF flow chart in each period is illustrated in Figure 4.

\subsection{Computational complexity analysis}

Computational complexity for an algorithm is an important index whether the algorithm provides processing capability in real time, and it is crucial for real-time systems. Hence, many filters with good performances in accuracy are limited in target tracking area due to their big computation. Here, computational complexity mainly denotes the times in terms of multiplications, divisions, additions, and subtraction for each run of an algorithm under no consideration of the process of generation, move, and measure for a target.

As described in Section 2, a state vector is assumed to be $n$-dimensional, and an observation vector is assumed to be $m$-dimensional, generally $m<n$. In addition, $M$ is the number of fuzzy rules utilized as mentioned in Section 4.1. The computational procedure for FLRLSF mainly consists of three steps: the update of the state, covariance, and fading factor corresponding to Equations 23, 24, and

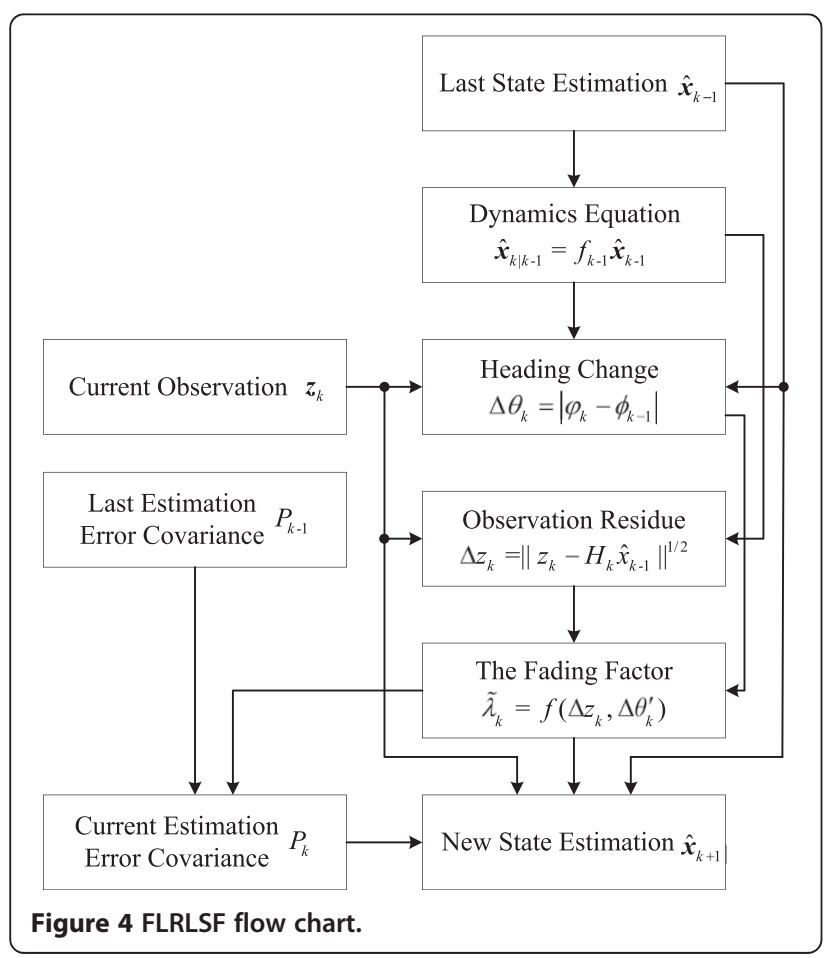

Table 2 Computational complexity for each filter

\begin{tabular}{ll}
\hline Filter & Computational complexity \\
\hline RLSF & $14 n^{3}+(4 m-1) n^{2}+\left(12 m^{2}+2 m-1\right) n+2 m^{3}-m^{2}+4$ \\
FLRLSF & $14 n^{3}+(4 m-1) n^{2}+\left(12 m^{2}+2 m-1\right) n+2 m^{3}-m^{2}+3 M+3$ \\
Fa-BF & $2 n^{2}+(2 m-1) n+3 M-1$ \\
HKF & $4 n^{3}+(12 m+6) n^{2}+\left(12 m^{2}+2\right) n+4 m^{3}+2 m^{2}-4 m+3 M$ \\
\hline
\end{tabular}

25 , respectively. The computation of three steps is $(4 m+$ 2) $n^{2}+(2 m-1) n, 14 n^{3}-3 n^{2}+\left(12 m^{2}-4 m\right) n+2 m^{3}-m^{2}+$ 4 , and $3 M-1$, respectively. As a result, the computational complexity for FLRLSF is $14 n^{3}+(4 m-1) n^{2}+\left(12 m^{2}+2\right.$ $m-1) n+2 m^{3}-m^{2}+3 M+3$. Similarly, the computational complexity for other three types of filters is shown in Table 2. Here, $M$ is related to the number of fuzzy rules for each filter, generally different with one another. From Table 2, the order of computational complexity for four filters from small to large is $F \alpha-\beta F$, RLSF, FLRLSF, and HKF, respectively.

\section{Experimental results and analysis}

A stimulation experiment and a real test experiment have been carried out to evaluate the performance of the FLRLSF method in comparison with the other three existing methods, the traditional RLSF [18], FA $\alpha-\beta F$ [16], and HKF [14] for MTT.

\subsection{Simulation experiment}

In the simulation experiment, single radar is assumed to track a maneuvering target, and the following assumptions and the radar performance parameters are used. The radar initial position is located at $(0 \mathrm{~m}, 0 \mathrm{~m})$, and its performance parameters are as follows: the system transmission delay $\tau=0 \mathrm{~s}$, sampling interval $T=5 \mathrm{~s}$, range error $\sigma_{r}=50 \mathrm{~m}$, and azimuth error $\sigma_{\beta}=0.01^{\circ}$. In the simulation scenario, the target moves in the air surveillance of 2-D Cartesian $x y$-plane according to the given

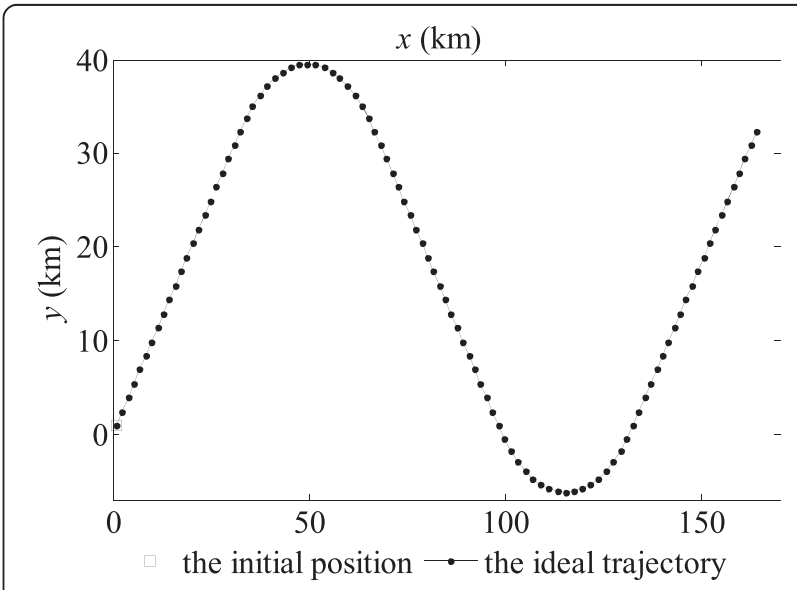

Figure 5 Ideal target trajectory in maneuver motion. 


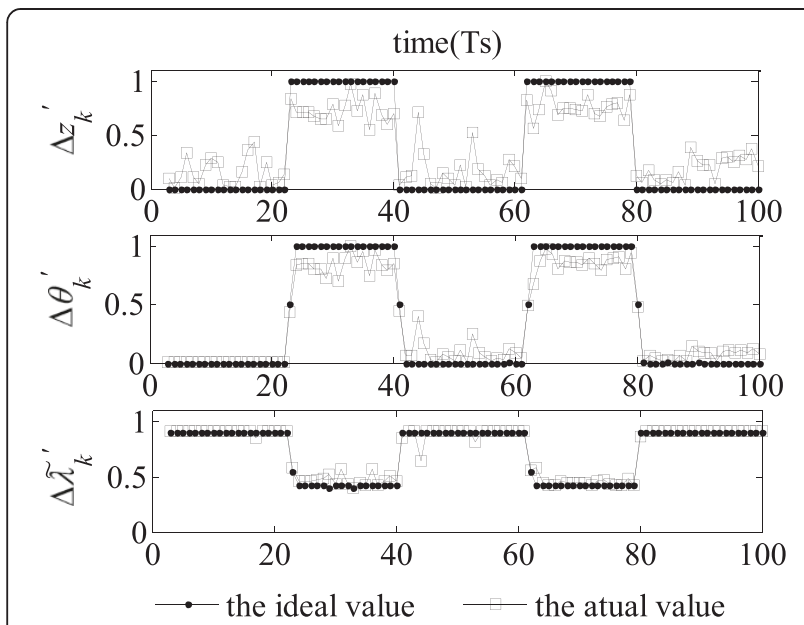

Figure $6 \Delta \theta_{k}{ }^{\prime}, \Delta z_{k}{ }^{\prime}$ and $\tilde{\lambda}_{k}$ estimate in maneuver motion.

trajectory below. Its initial state is given by $x_{0}=[800 \mathrm{~m}$, $200 \mathrm{~m} / \mathrm{s}, 900 \mathrm{~m}, 346 \mathrm{~m} / \mathrm{s}]^{\mathrm{T}}$, and its trajectory is formed by 100 observations collected by the radar, which is divided into five phases: constant velocity motion, turn rate motion $(0.2 \mathrm{rad} / \mathrm{s})$, constant velocity motion, turn rate motion $(-0.2 \mathrm{rad} / \mathrm{s})$, and constant velocity motion, as shown in Figure 5. The turn rate model of the target motion is characterized by the following equation:

$$
\boldsymbol{x}_{k}=\left[\begin{array}{cccc}
1 & \frac{\sin \omega_{k} T}{\omega_{k}} & 0 & -\frac{1-\cos \omega_{k} T}{\omega_{k}} \\
0 & \cos \omega_{k} T & 0 & -\sin \omega_{k} T \\
0 & \frac{1-\cos \omega_{k} T}{\omega_{k}} & 1 & \frac{\sin \omega_{k} T}{\omega_{k}} \\
0 & \sin \omega_{k} T & 0 & \cos \omega_{k} T
\end{array}\right] \boldsymbol{x}_{k-1}+\boldsymbol{v}_{k}
$$

where $\omega_{k}$ is the turn rate and $\boldsymbol{v}_{k}$ is the zero-mean white Gaussian noise with an unknown covariance. The simulation experiment is conducted by using a computer

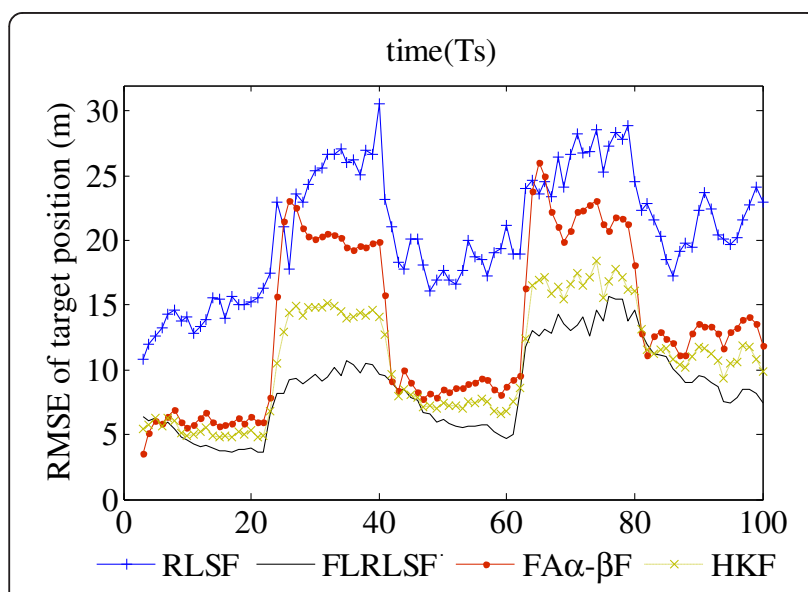

Figure 7 Estimate errors for four filters.

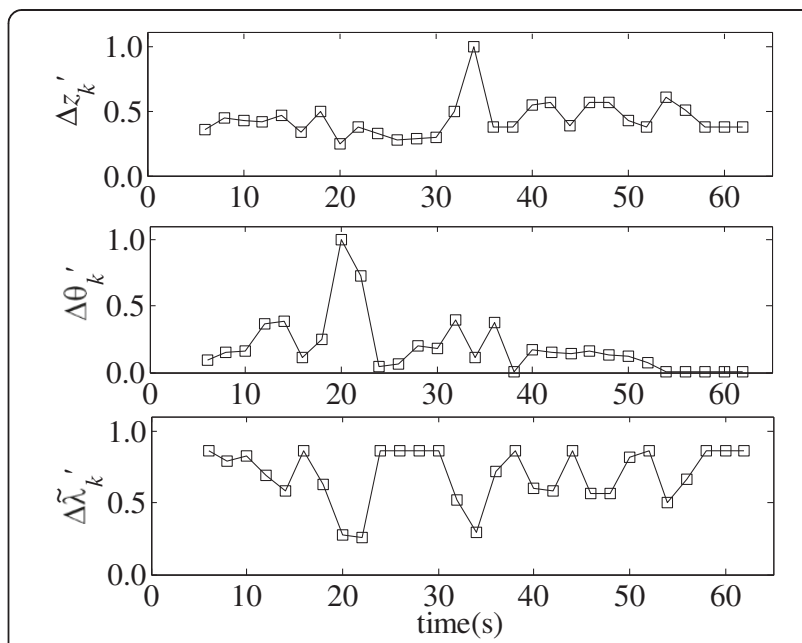

Figure $8 \Delta \theta_{k}{ }^{\prime} \Delta z_{k}{ }^{\prime}$, and $\tilde{\lambda}_{k}$ estimate in maneuver motion.

with a dual-core CPU of Pentium $42.93 \mathrm{GHz}, 1-\mathrm{GB}$ RAM. The programs are performed by using the Matlab 2009a version software.

Figure 6 shows that the variational curves of $\Delta z_{k}{ }^{\prime}, \Delta \theta_{k}{ }^{\prime}$, and $\tilde{\lambda}_{k}$ are obtained according to the target motion in the ideal (noiseless) and noisy case, respectively. Figure 7 provides the root mean squared errors (RMSE) for the target positions using three methods for 100 Monte Carlo simulation runs. As can be shown from Figure 6, it is verified that the curves of $\Delta z_{k}{ }^{\prime}$ and $\Delta \theta_{k}{ }^{\prime}$ are able to reflect the maneuver changes exactly, and $\tilde{\lambda}_{k}$ can also be adjusted dynamically with their magnitudes by using the fuzzy system in this case. It can be seen from Figure 7 that the RMSE of FLRLSF is smaller than those of RLSF, $F A \alpha-\beta F$, and HKF when the target moves at both constant velocity and turn rate, discussed as follows. Due to the perfect capability of FLRLSF to detect maneuver quickly and obtain its magnitude exactly, FLRLSF is able

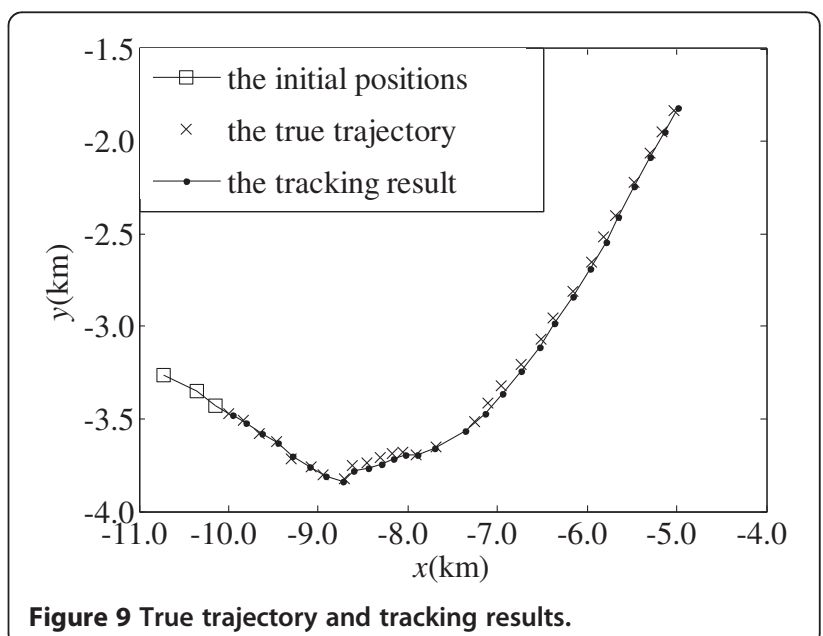


to estimate the target state timely and accurately. The average RMSE of the target positions by using FLRLSF, RLSF, FA $\alpha-\beta F$, and HKF are 8.8, 21.8, 13.5, and $10.8 \mathrm{~m}$ respectively, and the corresponding average run time of CPU is $0.0089,0.0083,0.0047$, and 0.0163 s. Hence, the results on average run time are consistent with the analysis for four filters in Section 4.3. Here, the average run time of FLRLSF is more than that of RLSF since the execution of the fuzzy system needs to consume a certain time. In addition, to compare with PF, the additional conditions are assumed to satisfy its run requirements [11]. The number of particles is given as 1,000 . The average run time for PF is $0.8632 \mathrm{~s}$, much longer than the four types of filters mentioned above.

\subsection{Real test experiment}

The real test experiment is evaluated by using real tracking data generated from some type of single radar. The radar performance parameters are represented as follows: the sampling interval $T=2 \mathrm{~s}$, maximum detection range $r_{\max }=21 \mathrm{~km}$, range error $\sigma_{r}=20 \mathrm{~m}$, and azimuth error $\sigma_{\beta}=0.5^{\circ}$. The initial position of the target is $z_{0}=$ $[800 \mathrm{~m}, 900 \mathrm{~m}]^{\mathrm{T}}$, and its trajectory is formed by $32 \mathrm{ob}-$ servations gathered by the radar. Figure 8 shows that the variational curves of $\Delta z_{k}^{\prime}, \Delta \theta_{k}^{\prime}$, and $\tilde{\lambda}_{k}$ are obtained according to the real tracking data. Figure 9 shows the real trajectory and the tracking results by using the proposed filter. The results in Figures 8 and 9 are analyzed below. Due to the rapid flight velocity, long flight time, and large azimuth error, the maneuver changes of the target are relatively complicated in the real test scenario. As can be known from Figure $8, \tilde{\lambda}_{k}$ is able to estimate the maneuver magnitudes based on $\Delta z_{k}{ }^{\prime}$ and $\Delta \theta_{k}{ }^{\prime}$. Because of the capability of a triangular function to suppress noise of the inputs, the proposed filter is still able to detect maneuvers exactly by using the fuzzy system. As can be seen from Figure 9, FLRLSF is able to track the maneuvering target accurately in the real situation. As a result, it is verified that the proposed filter is feasible in real MTT.

\section{Conclusion}

In this paper, considering the properties and drawbacks of the traditional adaptive filters, FLRLSF is proposed for MTT in the situation of observations with unknown random characteristics. The paper employs the fading factor of RLSF to describe maneuver characteristics of the motion model, analyzes the relationship of the fading factor with observation residuals and heading changes, and maps the relationship into a set of fuzzy rules. By applying fuzzy logic in the standard RLSF and designing the fuzzy system, the fading factor is allowed to be adjusted adaptively based on the fuzzy rules. These rules determine the fading factor dynamically according to the magnitudes of the observation residual and heading change in the current prediction. Therefore, FLRLSF is able to detect maneuvers timely and estimate their magnitude accurately. The effectiveness of the proposed filter is evaluated by using the simulation and real test experiment. Its performance in terms of tracking accuracy and the average run time is compared against those of RLSF, FA $\alpha-\beta F$, and HKF. The results of the simulation experiment show that the proposed filter can track a maneuvering target adaptively, and it achieves better performance in tracking accuracy than the other three methods. In addition, the real test experiment validates its feasibility in real environment.

In future work, we will extend the proposed filter in tracking multiple targets in cluster in distributed multisensor system. Furthermore, we will also explore FLRLSF for the state estimates of global tracks after local track-tolocal track fusion in the fusion center of the system.

\section{Competing interest}

The authors declare that they have no competing financial interests.

\section{Authors' information}

EF received his Bachelor's Degree in Electronic Information Science and Technology from Hubei Engineering University, Xiaogan in 2002 and Master's Degree in Signal and Information Processing from Nanchang Hangkong University, Nanchang in 2006. He is currently pursuing his PhD in Signal and Information Processing at Xidian University, Xi'an, China. His fields of interests include intelligent information processing and multisensor data fusion. WX graduated from Xidian University, Xi'an and remained at the university as a faculty member in 1965. From 1981 to 1983, he was a visiting scholar at the University of Pennsylvania, USA. In 1989, he was invited to the same university as a visiting professor. He is currently working in the School of Information Engineering, Shenzhen University, Shenzhen, China. His fields of interests include intelligent information processing, fuzzy information processing, image processing, pattern recognition, etc. ZL received his bachelor's degree and master's degree from Tianjin University, Tianjin in 1985 and 1990, respectively, and PhD degree from Xidian University, Xi'an in 2005. He is currently working in the School of Information Engineering, Shenzhen University, Shenzhen, China. His fields of interests include intelligent information processing, fuzzy information processing, and multisensor data fusion.

\section{Acknowledgements}

This work was supported by the National Natural Science Foundation of China (Grant: 61271107, 61301074), Key Project in the National Science \& Technology Pillar Program (2011BAH24B12), Specialized Research Fund for the Doctoral Program of Higher Education (20104408120001), and Natural Science Foundation of Ministry of Education (S2012010009417).

\section{Author details}

${ }^{1}$ School of Electronic Engineering, Xidian University, Xi'an, Shanxi 710071, China. ${ }^{2}$ ATR Key Laboratory, Shenzhen University, Shenzhen, Guangdong 518060, China.

Received: 20 December 2013 Accepted: 31 March 2014 Published: 16 April 2014

\section{References}

1. XR Li, VP Jilkov, A survey of maneuvering target tracking-part Vlc: approximate nonlinear density filtering in discrete time, in Proceedings of the SPIE Conference on Signal and Data Processing of Small Targets (CSDPST'12), vol. 8393 (SPIE, 2012), pp. 1-12 
2. Q Pan, Y Liang, F Yang, YM Cheng, Modern Target Tracking and Information Fusion (National Defense Industry Press, Beijing, 2009), pp. 5-61

3. XR Li, VP Jilkov, Survey of maneuvering target tracking. Part V: multiplemodel methods. IEEE Trans. Aero. Elec. Sys 41, 1255-1321 (2005)

4. N Nadarajah, R Tharmarasa, M Mcdonald, T Kirubarajan, IMM forward filtering and backward smoothing for maneuvering target tracking. IEEE Trans. Aero. Elec. Sys. 48(3), 2673-2678 (2012)

5. XR Li, VP Jilkov, Survey of maneuvering target tracking. Part l: dynamic models. IEEE Trans. Aero. Elec. Sys. 39, 1333-1364 (2003)

6. WY Chiu, BS Chen, Mobile location estimation in urban areas using mixed Manhattan/Eulidean norm and convex optimization. IEEE Trans. Wir. Commun. 8, 414-423 (2009)

7. WY Chiu, BS Chen, CY Yang, Robust relative location estimation in wireless sensor networks with inexact position problems. IEEE Trans. Mobile Comput. 11, 935-946 (2012)

8. WY Chiu, BS Chen, A mixed-norm approach using simulated annealing with changeable neighborhood for mobile location estimation. IEEE Trans. Mobile Comput. 9, 633-642 (2010)

9. S Sutharsan, T Kirubarajan, T Lang, M McDonald, An optimization based parallel particle filter for multitarget tracking. IEEE Trans. Aero. Elec. Sys. 48(2), 1601-1618 (2012)

10. LQ Li, WX Xie, Intuitionistic fuzzy joint probabilistic data association filter and its application to multitarget target tracking. Signal Process. 96, 433-444 (2014)

11. L Mihaylova, A Hegyi, A Gning, RK Boel, Parallelized particle and Gaussian sum particle filters for large-scale freeway traffic systems. IEEE Trans. Intell. Transp. Sys. 13(1), 36-48 (2012)

12. H Kim, I Kim, Design of adaptive fuzzy IMM algorithm for tracking the maneuvering target with time-varying measurement noise. Int. J. Control Autom. 5(3), 307-316 (2007)

13. D Jwo, S Wang, Adaptive fuzzy strong tracking extended Kalman filtering for GPS Navigation. IEEE Sens. J. 7(5), 778-789 (2007)

14. MH Barhari, A Karsaz, N Pariz, High maneuvering target tracking using a novel hybrid Kalman filter-fuzzy logic architecture. Int. J. Innov. Comput. 7, 501-510 (2011)

15. KCC Chan, V Lee, $H$ Leung, Radar tracking for air surveillance in a stressful environment using a fuzzy-gain filter. IEEE Trans. Fuzzy Syst. 5, 80-89 (1997)

16. $P F L i, J P Y u, L Q L i$, Maneuvering target tracking based on fuzzy adaptive $a-\beta$ filter. J. Syst. Eng. Electron. 30(11), 2138-2141 (2008)

17. YZ Zhu, Efficient recursive state estimator for dynamic systems without knowledge of noise covariances. IEEE Trans. Aero. Elec. Sys. 35, 102-114 (1999)

18. DL Peng, WC Lin, AK Xue, Multisensor Multisource Information Fusion Theory with Application (Science Press, Beijing, 2010), pp. 68-71

19. MH Bahari, MBN Sistani, N Pariz, Intelligent fading memory for high maneuvering target tracking. Int. J. Physic. Sci. 4, 548-554 (2009)

20. Y Liang, DH Zhou, L Zhang, Q Pan, Adaptive filtering for stochastic systems with generalized disturbance inputs. IEEE Signal Proc. Let. 15, 645-648 (2008)

21. LX Wang, Adaptive Fuzzy Systems and Control: Design and Stability Analysis (National Defense Industry Press, Beijing, 1995), pp. 210-232

22. XJ Su, P Shi, YD Song, A novel approach to filter design for T-S fuzzy discrete-time systems with time-varying delay. IEEE Trans. Fuzzy Syst. 20, 1114-1129 (2012) 